\title{
Escala de diagnóstico (SI5) de disfunción de la articulación sacroiliaca: estudio piloto
}

\author{
J. C. Acevedo González y y S. T. Quintero² \\ ${ }^{1}$ Unidad de Neurocirugía. Hospital Universitario San Ignacio. Facultad de Medicina Pontificia Universidad \\ Javeriana. Bogotá, Colombia. ${ }^{2}$ Unidad de Neurocirugía. Facultad de Medicina Pontificia Universidad Jave- \\ riana. Hospital Universitario San Ignacio. Bogotá, Colombia
}

Acevedo González JC, Quintero ST. Escala de diagnóstico (SI5) de disfunción de la articulación sacroiliaca: estudio piloto. Rev Soc Esp Dolor 2014; 21(3): 123-130.

\begin{abstract}
Objective: Low back pain is one of the most frequent symptoms in the general population. The sacroiliac joint dysfunction is a known cause of low back pain. Some studies have found that the clinical characteristic and physical examination findings are consistently capable of identifying dysfunctional sacroiliac joint as pain generators. However many factors can impact on the sensitivity and specificity of diagnostic. In this context, we think that a test score (SI5) may be performed to assess diagnostic utility of clinical signs and radionuclide bone scanning of sacroiliac joint dysfunction. The primary aim of the present study was to conduct conducting the pilot study of our new test score SI5.

Methods: After reviewing the literature about of clinical characteristic, diagnostic test and imaging of the sacroiliac joint; we evaluated diagnostic utility of these aspects and developed the SI5 test score. The SI 5 test score was performed in 68 patients with low back pain including completion of a standard questionnaire, history, physical examination performed by a physician, and evaluation of the results. Patients included in this study were assigned to one of four groups: A group of pain from facet joints, the second group with radicular pain, others patients
\end{abstract}

Financiación: Hospital Universitario San Ignacio - Pontificia Universidad Javeriana. Bogotá, Colombia.

Recibido: 01-09-13.

Aceptado: 07-12-13. were designed in the sacroiliac pain group and last group with other causes of low back pain.

Results: All patients with diagnostic of sacroiliac joint dysfunction had a SI5 test score SI5 of 4 to 5; instead the patients in the other groups had less than 4 point in the test score. The frequency of all criteria of the SI5 test score was higher in the patients of sacroiliac pain group than in the other groups.

Discussion and conclusions: Sacroiliac joints have been shown to be capable of producing pain in the low back; however, the diagnostic value of examination tests for sacroiliac joint pain has been questioned previously by other authors. More research is needed to guide clinicians on the choice of examination procedures and interventions for patients with pain which may be arising from the sacroiliac joint. The pilot study of SI5 test score provides an opportunity to gather both quantitative and qualitative data about the test. This study required statistical procedures that yield appropriate results.

Key words: Sacroiliac joint. Low back pain. Pilot study.

\section{RESUMEN}

Objetivo: El dolor lumbar es uno de los síntomas más frecuentes en la población general. La disfunción biomecánica de la articulación sacroiliaca (ASI) es una de las etiologías que debe considerarse en el estudio de esta patología. En este contexto consideramos que el análisis clínico de la ASI debe permitir desarrollar una escala para confirmar el diagnóstico de su disfunción, sin que sea necesaria la realización de procedimientos invasivos. El presente trabajo es un estudio piloto de la aplicación de la escala diagnóstica para la disfunción de la ASI (SI5).

Métodos: Se desarrolló una búsqueda bibliográfica de los signos clínicos y paraclínicos comúnmente utilizados para sospechar disfunción de la ASI. Una vez se discutieron y se definieron los aspectos más importantes, se desarrolló la escala de diagnóstico de la disfunción de la ASI (SI5). Se aplicó la escala SI5 a 68 pacientes que ingresaron con dolor lumbar crónico mediante un cuestionario estandarizado. Se establecieron cuatro grupos de pacientes: grupo 1 - pacientes con enfermedad 
Rev. Soc. Esp. del Dolor, Vol. 21, N. ${ }^{\circ}$ 3, Mayo-Junio 2014

facetaria lumbar; grupo 2 - pacientes con dolor neuropático de origen radicular; grupo 3 - pacientes con diagnóstico de disfunción de la ASI; y grupo 4 - pacientes con otras causas de dolor lumbar (en este grupo se incluyeron aquellos con dolor lumbar discogénico y dolor osteomuscular).

Resultados: Al aplicar la Escala de Disfunción de la ASI (SI5) en los cuatro grupos descritos encontramos que todos los pacientes con diagnóstico de disfunción de la ASI (grupo 3) tienen cuatro puntos o más en la escala. Por el contrario en los demás grupos la mayoría de los pacientes tuvieron menos de cuatro puntos en la escala SI5. Los cinco aspectos evaluados fueron más frecuentemente positivos en los pacientes con diagnóstico de disfunción de la ASI comparados con los demás grupos.

Discusión y conclusiones: La disfunción de la articulación sacroiliaca es una patología importante como causa de dolor lumbar, sin embargo su diagnóstico clínico es muy difícil, por lo cual se hace necesario definir una combinación de test clínicos y de estudios paraclínicos para lograr diagnósticos más precisos. Sin embargo consideramos que se requiere un proceso estadístico completo de validación de la Escala SI5. Nuestros resultados son un estudio piloto inicial que nos va a permitir estructurar y redefinir una Escala de Disfunción de la ASI para concluir con mayor certeza.

Palabras clave: Articulación sacroiliaca. Dolor lumbar. Estudio piloto.

\section{INTRODUCCIÓN}

El dolor lumbar es uno de los síntomas más frecuentes en la población general. Algunos autores refieren que hasta el $50 \%$ de los adultos ha presentado un episodio de dolor lumbar en algún momento de su vida. Según las Guías Europeas para el manejo del dolor lumbar crónico no específico la prevalencia de vida de dolor lumbar es de $84 \%$ (1). Este dolor puede ocasionar un impacto adverso sobre la calidad de vida de los pacientes y un alto ausentismo laboral, lo cual ocasiona una problemática socioeconómica importante. Esto conlleva a que las sociedades modernas tengan la necesidad de realizar diagnósticos precisos y tratamientos efectivos para este tipo de patologías $(1,2)$.

La mayor dificultad para determinar la etiología del dolor lumbar radica en que la columna vertebral y la pelvis funcionan como una estructura dinámica integrada. Cualquier modificación de una estructura lumbosacrococcígea repercute directamente en los discos y las articulaciones de los niveles vertebrales, por lo cual hay diversas estructuras anatómicas que podrían estar implicadas en el origen de este tipo de dolor (3-5). Por todo lo anterior los médicos que tratamos este tipo de patologías reconocemos que los diagnósticos diferenciales al considerar el dolor lumbar son numerosos y de difícil identificación específica.

La disfunción biomecánica de la articulación sacroiliaca (ASI) es una de las patologías que debe considerarse en el estudio de pacientes con dolor lumbar $(3,6-29)$.
En varios estudios se ha reportado una prevalencia de la disfunción de la articulación sacroiliaca en 10-30 \% en paciente con dolor lumbar (12,30-34). Esto conduce al desarrollo de múltiples elementos clínicos y paraclínicos para diagnosticar el dolor que se origina en la ASI, sin embargo actualmente no contamos con unos criterios claros y prácticos para aplicar en la consulta. Muchos signos clínicos se han descrito pero se desconoce cuáles son los más eficaces y los que permiten una mayor especificidad y sensibilidad diagnóstica $(5,6,35,36)$. La Asociación Internacional para el Estudio del Dolor (IASP) desarrolló criterios diagnósticos en donde de manera inespecífica considera que cualquier signo positivo de provocación dolorosa puede ser suficiente para considerar el diagnóstico de disfunción de la articulación sacroiliaca. De la misma forma se incluye entre los criterios la realización de un bloqueo selectivo de la articulación con mejoría clínica definitiva o transitoria (30-33). Esto implica que debe realizarse un procedimiento invasivo para hacer el diagnóstico.

A partir de la importancia del diagnóstico de la disfunción de la articulación sacroiliaca, muchos autores han tratado de establecer la especificidad y sensibilidad de los diversos aspectos del dolor lumbar para diagnosticar esta patología. Adicionalmente se han combinado diversos signos y síntomas para aumentar la probabilidad de un diagnóstico correcto $(32,37)$. En paralelo la estadística durante su desarrollo ha resaltado la necesidad de medir las variables que implican un importante grado de subjetividad para establecer datos más objetivos, entre estos el dolor lumbar y sus características asociadas.

Las características del dolor lumbar tienen múltiples aspectos difíciles de cuantificar, el estudio de esta, y de muchas otras patologías, ha generado mucho interés en investigación clínica y a partir de esto se han desarrollado métodos que solucionan estas dificultades de evaluación. La complejidad en la medición de este tipo de variables radica en la dificultad para estandarizar el proceso de evaluación y de repetibilidad, por lo cual se han establecido estrategias especiales como la adaptación y validación de escalas con el objetivo de medir dichas variables.

En este contexto consideramos que el análisis clínico de la articulación debe permitir desarrollar una escala para confirmar el diagnóstico de dolor en la ASI sin que sea necesaria la realización de procedimientos invasivos. El presente trabajo es un estudio piloto de la aplicación de la escala diagnóstica para la disfunción de la ASI (SI5).

\section{MATERIALES Y MÉTODOS}

- Búsqueda de información: se realizó una búsqueda de artículos en inglés, francés y español de todos los signos clínicos y paraclínicos comúnmente utilizados para sospechar disfunción de la ASI en Medline, 
PubMed, Science Direct, Ovid y Hinari. Se encontraron 62 artículos enfocados en el diagnóstico de dolor lumbar y su correlación con la disfunción de la ASI. De estos artículos se obtuvieron las características clínicas de disfunción en la articulación sacroiliaca. Se identificaron 40 signos clínicos que sugieren disfunción de esta articulación. Se estableció además la utilidad de las imágenes diagnósticas en la patología de la ASI; la gammagrafía ósea fue considerada en el estudio con mayor utilidad para diagnóstico de esta patología.

- Evaluación de la importancia de los aspectos evaluados: se realizó un listado de todos los signos y síntomas encontrados, al igual que exámenes paraclínicos para diagnóstico de disfunción de la ASI. Se evaluaron según la sensibilidad y la especificidad encontrada en los diferentes estudios, se consideraron más importantes aquellos signos que fueron evaluados por varios estudios con resultados similares.

- Definición de la escala propuesta: una vez se discutieron y se definieron los aspectos clínicos y paraclínicos más importantes para lograr el diagnóstico de disfunción de la ASI, se desarrolló la escala de diagnóstico de la disfunción de la ASI (SI5) que evalúa cinco aspectos (Tabla I).

- Diseño del instrumento: se diseñó un cuestionario estandarizado para evaluar los cinco aspectos de la escala (Tabla II). La primera parte del cuestionario evalúa aspectos clínicos característicos del dolor lumbar secundario a la disfunción de la ASI. Se evaluó por medio de preguntas autoadministradas por el paciente o sus familiares. Debían establecer si presen- taban o no las cuatro características del dolor lumbar asociadas a disfunción de la ASI. La segunda parte fue desarrollada por el médico tratante, se asignó un punto por cada signo positivo. En la última parte del cuestionario se registró si la gammagrafía ósea reportaba índice sacroiliaco elevado. Se dio un punto adicional si el índice sacroiliaco fue mayor 1,5 (Tabla II).

- Aplicación del instrumento: se aplicó este cuestionario a todos los pacientes que ingresaron a tratamiento percutáneo para manejo de dolor. Fue diligenciado previo a la realización del procedimiento indicado.

- Tratamiento percutáneo: a todos los pacientes a quienes se les aplicó el cuestionario se les realizó el bloqueo percutáneo que se indicó por su neurocirujano tratante. Dos semanas después del procedimiento se realizó una consulta de control donde se evaluó la respuesta al tratamiento.

- Evaluación de resultados: se aplicó el cuestionario a 68 pacientes que ingresaron con dolor lumbar crónico con indicación por médicos tratantes de tratamiento percutáneo para manejo del dolor. Fueron $62 \%$ (42) del género femenino y sólo $38 \%$ (26) hombres. La edad promedio fue 57 años con un intervalo entre 18-84 años.

Según las características clínicas, examen físico y estudios imageneológicos, se establecieron cuatro grupos de pacientes: grupo 1 - pacientes con enfermedad facetaria lumbar; grupo 2 - pacientes con dolor neuropático de origen radicular; grupo 3 - pacientes con diagnóstico de disfunción de la ASI; y grupo 4 - pacientes con otras causas de dolor lumbar (en este grupo se incluyeron aquellos con dolor lumbar discogénico y dolor osteomuscular) (Tabla III).

TABLA I. ESCALA DE DIAGNÓSTICO DE DISFUNCIÓN DE LA ARTICULACIÓN SACROILIACA (SI5)

\begin{tabular}{|c|c|c|}
\hline Aspecto evaluado & \multicolumn{2}{|c|}{ Puntaje } \\
\hline $\begin{array}{l}\text { 1. Dolor localizado en la región lumbar (incluyendo glúteos) con } \\
\text { características mecánicas }\end{array}$ & $\mathrm{POS}=1$ & $\mathrm{NEG}=0$ \\
\hline \multicolumn{3}{|c|}{$\begin{array}{l}\text { Para ser positivo debe tener al menos } 3 \text { de } 4 \text { de los siguientes elementos: } \\
\text { - Dolor que mejora con el reposo. Al estar en decúbito sin moverse, el dolor disminuye al menos un } 50 \% \\
\text { - Dolor que aparece con el movimiento en la cama. Al estar en decúbito si el paciente desea cambiar de posición } \\
\text { aparece dolor sobre la región lumbar } \\
\text { - Dolor que aparece estando sentado al levantarse de la silla. El paciente estando sentado en una silla siente dolor al } \\
\text { despegar las nalgas de la superficie horizontal de la silla } \\
\text { - Dolor que aparece con las maniobras de movilización de la columna lumbosacra y/o coccígea, principalmente en la } \\
\text { extensión }\end{array}$} \\
\hline 2. Número 2: Test de Yeomans & $\mathrm{POS}=1$ & $\mathrm{NEG}=0$ \\
\hline 3. Número 3: Test de Patrick & $\mathrm{POS}=1$ & $\mathrm{NEG}=0$ \\
\hline 4. Número 4: Test de Gaenslen & $\mathrm{POS}=1$ & $\mathrm{NEG}=0$ \\
\hline 5. Número 5: índice sacroiliaca en la gammagrafía ósea superior a 1,5 & $\mathrm{POS}=1$ & $\mathrm{NEG}=0$ \\
\hline
\end{tabular}

POS: positivo; NEG: negativo. 
TABLA II. CUESTIONARIO DE APLICACIÓN DE ESCALA DIAGNÓSTICA DE DISFUNCIÓN DE LA ARTICULACIÓN SACROILIACA

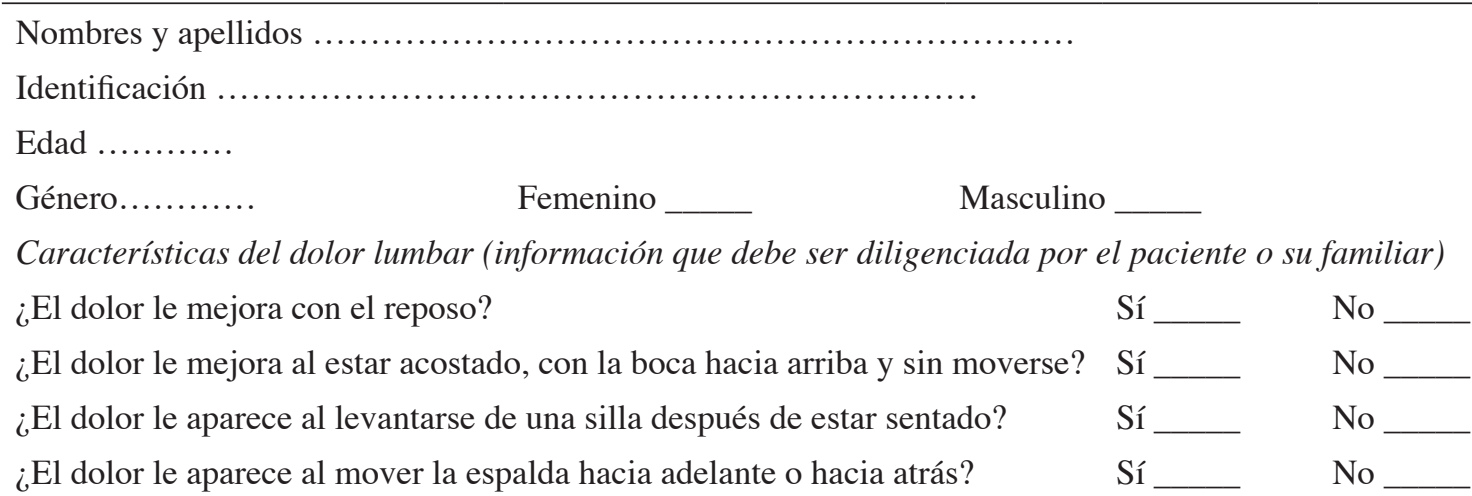

\section{Examen clínico (información que debe ser diligenciada por médico tratante)}

Test de Yeomans

POS

NEG

Test de Patrick

POS

NEG

Test de Gaenslen

POS

NEG

2. Apoyo diagnóstico (información que debe ser diligenciada por médico tratante)

Índice sacroiliaca en la gammagrafia ósea superior a 1,5

Sí _ No _

POS: positivo; NEG: negativo.

En cada grupo aplicamos la escala SI5 mediante el cuestionario estandarizado (Tabla II). El puntaje mínimo fue cero y el máximo 5 puntos. Todos los datos fueron registrados en una base de datos Excel 2010 para posterior análisis. Se evaluaron de manera descriptiva los resultados en los cuatro grupos de pacientes (Fig. 1).

\section{RESULTADOS}

$\mathrm{Al}$ evaluar los resultados de la aplicación de la Escala de Disfunción de la ASI (SI5) en los cuatro grupos descritos encontramos que todos los pacientes con diagnóstico de disfunción de la ASI (grupo 3) tienen cuatro puntos o

TABLA III. PACIENTES CON DOLOR LUMBAR CRÓNICO ESPECÍFICO, AGRUPADOS SEGÚN LAS CARACTERÍSTICAS CLÍNICAS, EXAMEN FÍSICO Y ESTUDIOS IMAGENEOLÓGICOS

\begin{tabular}{lc}
\hline Grupo de pacientes & Número \\
\hline Enfermedad facetaria & 33 \\
Radiculopatía & 25 \\
Disfunción de la ASI & 6 \\
Otras causas & 4 \\
\hline Total & 68 \\
\hline
\end{tabular}

más en la escala. Por el contrario en los demás grupos la mayoría de los pacientes tuvieron menos de cuatro puntos en la escala SI5 (Fig. 2).

El dolor lumbar característico (definido como tres de cuatro características) fue positivo en la gran mayoría de pacientes en casi todos los grupos ( $>80 \%$ en el grupo de enfermedad facetaria, radiculopatía y disfunción de la ASI).

Todos los pacientes con diagnóstico de disfunción de la ASI tuvieron test de Gaenslen positivo. En los demás grupos este test fue positivo en menos del $50 \%$ de los pacientes. El test de Yeomans y Patrick también fueron positivos en todos los pacientes del grupo de disfunción de la articulación sacroiliaca, en los demás grupos fue positiva en menos del $70 \%$ de los pacientes en cada grupo.

Solo ocho pacientes tuvieron índice sacroiliaco elevado $(>1,5)$, tres de estos tuvieron diagnóstico de disfunción de la ASI, los otros cinco pacientes tuvieron diagnóstico de enfermedad facetaria (3 pacientes) y radiculopatía (2 pacientes).

\section{DISCUSIÓN}

La región lumbosacra (RL) corresponde al área comprendida entre la última costilla en la parte superior, los glúteos en la parte inferior y hasta la porción más lateral de la espalda. Esta región incluye múltiples estructuras óseas, articulares, cartilaginosas, musculares, ligamentarias y nerviosas, que permiten el funcionamiento adecuado de esta 


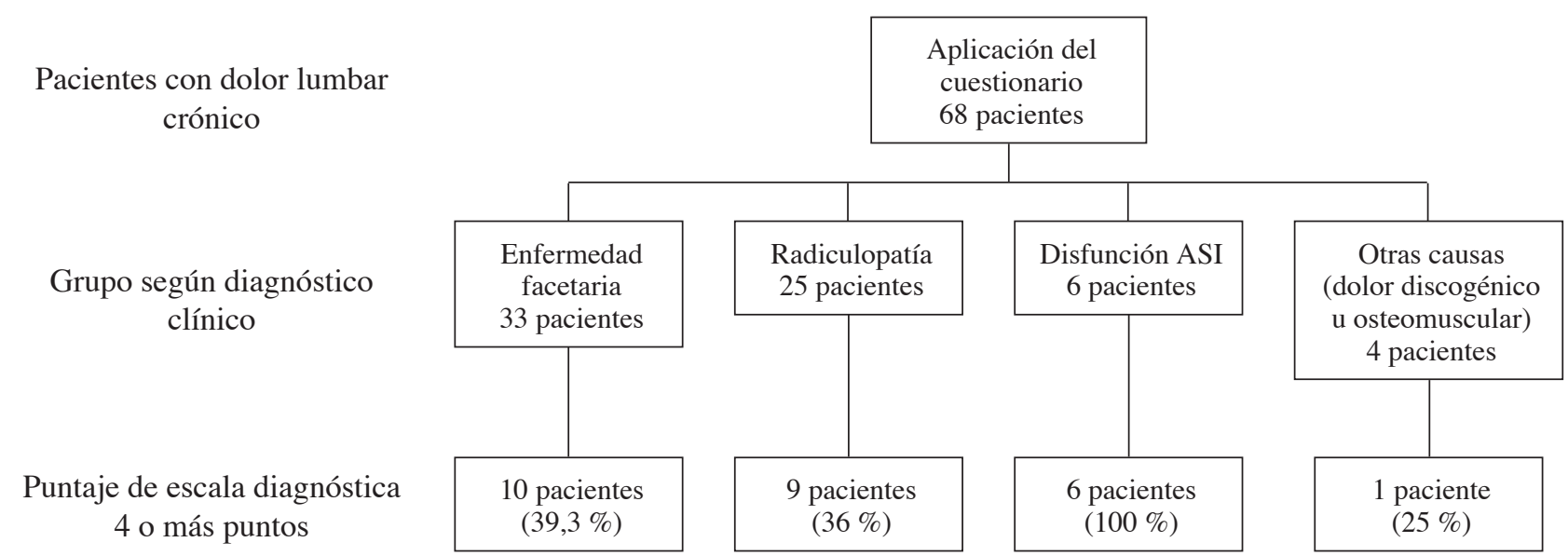

Fig. 1. Resultados de la aplicación de la escala diagnóstica de la disfunción de la articulación sacroiliaca (SI5) en pacientes con dolor lumbar crónico específico agrupados según diagnóstico clínico.

región del cuerpo que se somete a diario al mayor esfuerzo biomecánico. La interacción adecuada de cada una de estas estructuras así como su integridad anatómica permiten en el individuo un desempeño diario normal para una actividad física y psicológica adaptada a su edad y a sus características morfométricas.

La enfermedad facetaria ha sido reportada como la causa más frecuente de dolor lumbar por muchos autores (38-45). Sin embargo la ASI es una de las estructuras de mayor importancia en esta región.

Múltiples estudios clínicos han tratado de demostrar que la historia clínica y los hallazgos al examen físico son capaces de identificar la disfunción de la ASI como generadora de dolor. Dentro de la valoración clínica se han establecido múltiples aspectos del dolor originado en esta articulación, sin embargo no hay un consenso de las características comunes $(17,19,30,46,47)$. En nuestro estudio la mayoría de pacientes con dolor lumbar presenta las características de dolor de disfunción de la articulación sacroiliaca en todos los grupos de pacientes (> $80 \%$ ).

Para el diagnóstico de la disfunción de la ASI encontramos en la literatura 40 signos clínicos. Dentro de los diversos estudios los test de provocación de dolor se han considerado más confiables que otros tipos de test (nivel de evidencia B) $(1,30,32,33,37,48-55)$. Se ha determinado que hasta un $20 \%$ de los adultos asintomáticos tienen hallazgos clínicos positivos al desarrollar los test de provocación sacroiliaca y mientras algunos estudios han encontrado adecuada concordancia interevaluadores otros estudios no lo demuestran.

Después de la búsqueda en la literatura consideramos que de los más comunes test clínicos para diagnóstico de disfunción de la articulación sacroiliaca son el test de Patrick (sensibilidad 63-100 \% y especificidad 73-77 \%), el test de Gaenslen (sensibilidad $71 \%$ y especificidad $26 \%$ ) y el test de Yeomans por lo cual fueron incluidos en nuestro estudio $(1,30,32,56)$. En nuestro estudio todos los pacientes con diagnóstico de disfunción de la ASI tienen los test positivos.

El patrón característico de dolor ha sido otro objetivo de múltiples estudios, diversos investigadores han logrado reproducir el dolor en sujetos normales y han encontrado característicamente un dolor en región medial del muslo inferior a la espina iliaca posterosuperior, algunos sujetos pueden referir irradiación a región alta del muslo (30). Nuestro estudio evaluó características del dolor más que la región dolorosa, la localización del dolor según diversos estudios pueden aumentar la posibilidad de un diagnóstico correcto.

Los estudios imageneológicos de la articulación sacroiliaca han sido objeto de estudio para múltiples autores, la

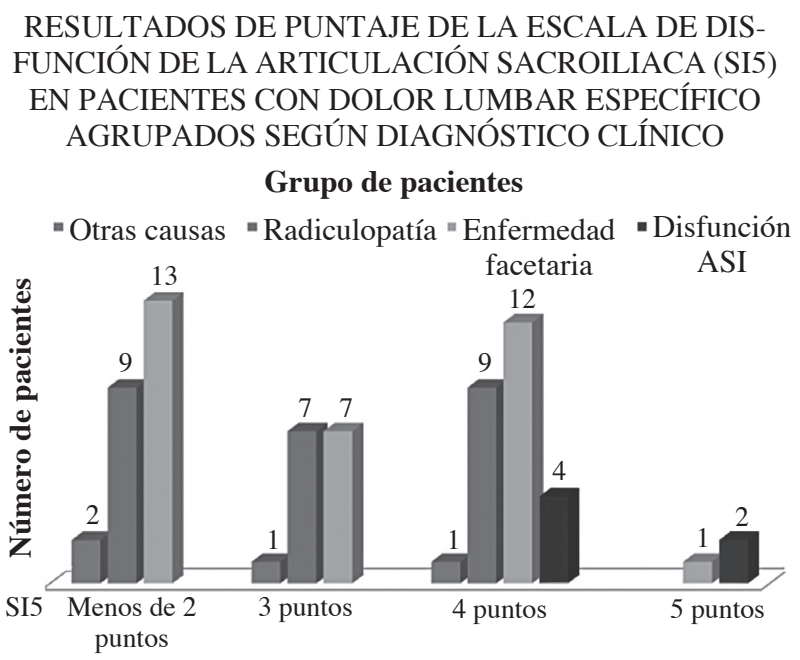

Fig. 2. Distribución de los resultados por grupos. Aplicación de la escala diagnóstica de la disfunción de la articulación sacroiliaca (SI5) en pacientes con dolor lumbar crónico específico. 
Rev. Soc. Esp. del Dolor, Vol. 21, N. ${ }^{\circ}$ 3, Mayo-Junio 2014

tomografía y la resonancia magnética de la articulación son métodos excelentes para detectar una lesión en la ASI, sin embargo muestra limitada utilidad para identificar cambios degenerativos, ya que en sujetos asintomáticos puede mostrar cambios hasta en el $100 \%$ de pacientes después de los 50 años. Por esta razón no lo consideramos un aspecto a evaluar en nuestro estudio $(6,14,57)$. La gammagrafía ósea contrario a lo anterior ha sido considerada un estudio útil en el diagnóstico de la disfunción de la ASI, con baja sensibilidad 13-46\% pero alta especificidad 89,5-100\% $(30,58)$; esta última característica es un aspecto importante para establecer el diagnóstico de esta patología, por lo cual fue incluida en nuestra escala diagnóstica. En nuestro estudio encontramos la gammagrafía con hipercaptación sacroiliaca en el $50 \%$ de los pacientes con diagnóstico de disfunción de la articulación.

En la literatura revisada se recomienda el bloqueo sacroiliaco como manejo inicial del paciente con hallazgos por historia clínica y al examen físico que sugieran disfunción de la articulación sacroiliaca. En caso de no lograr mejoría, se indicaría un bloqueo de las articulaciones facetarias, que por muchos autores representan la causa más frecuente de dolor lumbar específico y el diagnóstico diferencial más cercano (31). La denervación por radiofrecuencia de la articulación sacroiliaca ha mostrado utilidad para manejo del dolor de forma más prolongada, sin embargo la mayoría de autores la realizan posterior a establecer diagnóstico con un bloqueo intraarticular inicial (12,35,59-62).

\section{CONCLUSIÓN}

La historia clínica y el examen físico de un paciente con dolor lumbar son el abordaje inicial para establecer el diagnóstico de la etiología. La disfunción de la articulación sacroiliaca es una patología importante como causa de dolor lumbar, sin embargo su diagnóstico clínico es muy difícil, por lo cual se hace necesario definir una combinación de test clínicos y de estudios paraclínicos para lograr diagnósticos más precisos. Sin embargo consideramos que se requiere un proceso estadístico completo de validación de la Escala SI5. Nuestros resultados son un estudio piloto inicial que nos va a permitir estructurar y redefinir una Escala de Disfunción de la ASI para concluir con mayor certeza.

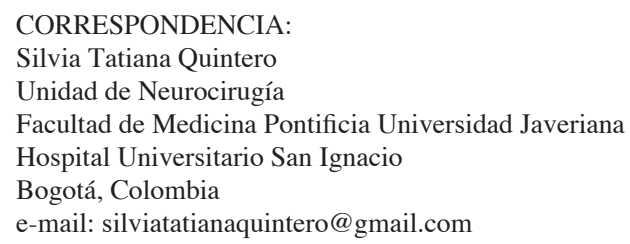

\section{BIBLIOGRAFÍA}

1. Airaksinen O, Brox JI, Cedraschi C, Hildebrandt J, KlaberMoffett J, Kovacs F, et al. Chapter 4. European guidelines for the management of chronic nonspecific low back pain. Eur Spine J 2006;15(Supl. 2):S192-300.

2. Bogduk N. Low back pain. Aust Fam Physician 1985;14(11):1168-70, 72.

3. Vleeming A, Schuenke MD, Masi AT, Carreiro JE, Danneels L, Willard FH. The sacroiliac joint: An overview of its anatomy, function and potential clinical implications. J Anat 2012;221(6):537-67.

4. Young S, Aprill C, Laslett M. Correlation of clinical examination characteristics with three sources of chronic low back pain. Spine J 2003;3(6):460-5.

5. Acevedo JC. Síndrome facetario lumbar. Nuevo signo de diagnóstico clínico. Rehabilitación (Madr) 2004;38(4):168-74.

6. Ha KY, Lee JS, Kim KW. Degeneration of sacroiliac joint after instrumented lumbar or lumbosacral fusion: A prospective cohort study over five-year follow-up. Spine (Phila Pa 1976) 2008;33(11):1192-8.

7. Vilensky JA, O'Connor BL, Fortin JD, Merkel GJ, Jimenez AM, Scofield BA, et al. Histologic analysis of neural elements in the human sacroiliac joint. Spine (Phila Pa 1976) 2002;27(11):1202-7.

8. Brown MF, Hukkanen MV, McCarthy ID, Redfern DR, Batten JJ, Crock HV, et al. Sensory and sympathetic innervation of the vertebral endplate in patients with degenerative disc disease. J Bone Joint Surg Br 1997;79(1):147-53.

9. McGrath MC, Zhang M. Lateral branches of dorsal sacral nerve plexus and the long posterior sacroiliac ligament. Surg Radiol Anat 2005;27(4):327-30.

10. McLain RF, Pickar JG. Mechanoreceptor endings in human thoracic and lumbar facet joints. Spine (Phila Pa 1976) 1998;23(2):168-73.

11. McLain RF. Mechanoreceptor endings in human cervical facet joints. Spine (Phila Pa 1976) 1994;19(5):495-501.

12. Yin W, Willard F, Carreiro J, Dreyfuss P. Sensory stimulation-guided sacroiliac joint radiofrequency neurotomy: Technique based on neuroanatomy of the dorsal sacral plexus. Spine (Phila Pa 1976) 2003;28(20):2419-25.

13. Fortin JD, Washington WJ, Falco FJ. Three pathways between the sacroiliac joint and neural structures. AJNR Am J Neuroradiol 1999;20(8):1429-34.

14. Malghem J, Vande Berg B, Lecouvet F, Koutaissoff S, Maldague B. Principles of analysis for sacroiliac joints imaging. JBR-BTR 2007;90(5):358-67.

15. Bakland O, Hansen JH. The "axial sacroiliac joint". Anat Clin 1984;6(1):29-36.

16. Bechtel R. Physical characteristics of the axial interosseous ligament of the human sacroiliac joint. Spine J 2001;1(4):255-9.

17. Fortin JD, Kissling RO, O’Connor BL, Vilensky JA. Sacroiliac joint innervation and pain. Am J Orthop (Belle Mead NJ) 1999;28(12):687-90.

18. Fortin JD, Aprill CN, Ponthieux B, Pier J. Sacroiliac joint: Pain referral maps upon applying a new injection/arthrography technique. Part II: Clinical evaluation. Spine (Phila Pa 1976) 1994;19(13):1483-9.

19. Grob KR, Neuhuber WL, Kissling RO. Innervation of the sacroiliac joint of the human. Z Rheumatol 1995;54(2):11722.

20. Harrison DE, Harrison DD, Troyanovich SJ. The sacroiliac joint: A review of anatomy and biomechanics with clinical 
implications. J Manipulative Physiol Ther 1997;20(9):60717.

21. Ilaslan H, Arslan A, Koc ON, Dalkilic T, Naderi S. Sacroiliac joint dysfunction. Turk Neurosurg 2010;20(3):398-401.

22. Jaovisidha S, Ryu KN, De Maeseneer M, Haghighi P, Goodwin D, Sartoris DJ, et al. Ventral sacroiliac ligament. Anatomic and pathologic considerations. Invest Radiol 1996;31(8):532-41.

23. Laban MM. Re: Sacroiliac joint pain: Anatomy, biomechanics, diagnosis, and treatment. Am J Phys Med Rehabil 2007;86(12):1032-3; author reply 3.

24. Murata Y, Takahashi K, Ohtori S, Moriya H. Innervation of the sacroiliac joint in rats by calcitonin gene-related peptideimmunoreactive nerve fibers and dorsal root ganglion neurons. Clin Anat 2007;20(1):82-8.

25. O'Shea FD, Boyle E, Salonen DC, Ammendolia C, Peterson $\mathrm{C}, \mathrm{Hsu} \mathrm{W}$, et al. Inflammatory and degenerative sacroiliac joint disease in a primary back pain cohort. Arthritis Care Res (Hoboken). 2010;62(4):447-54.

26. Vrahas M, Hern TC, Diangelo D, Kellam J, Tile M. Ligamentous contributions to pelvic stability. Orthopedics 1995;18(3):271-4.

27. Weksler N, Velan GJ, Semionov M, Gurevitch B, Klein M, Rozentsveig V, et al. The role of sacroiliac joint dysfunction in the genesis of low back pain: The obvious is not always right. Arch Orthop Trauma Surg 2007;127(10):885-8.

28. Yamashita T, Minaki Y, Oota I, Yokogushi K, Ishii S. Mechanosensitive afferent units in the lumbar intervertebral disc and adjacent muscle. Spine (Phila Pa 1976) 1993;18(15):2252-6.

29. Yong-Hing K. Sacro-iliac joint pain: Etiology and conservative treatment. Chir Organi Mov 1994;79(1):35-45.

30. Cohen SP. Sacroiliac joint pain: A comprehensive review of anatomy, diagnosis, and treatment. Anesth Analg 2005;101(5):1440-53.

31. Boswell MV, Trescot AM, Datta S, Schultz DM, Hansen $\mathrm{HC}$, Abdi S, et al. Interventional techniques: Evidencebased practice guidelines in the management of chronic spinal pain. Pain Physician 2007;10(1):7-111.

32. Hansen HC, McKenzie-Brown AM, Cohen SP, Swicegood JR, Colson JD, Manchikanti L. Sacroiliac joint interventions: a systematic review. Pain Physician 2007;10(1):16584.

33. Szadek KM, van der Wurff P, van Tulder MW, Zuurmond WW, Perez RS. Diagnostic validity of criteria for sacroiliac joint pain: A systematic review. J Pain 2009;10(4):354-68.

34. Dreyfuss P, Snyder BD, Park K, Willard F, Carreiro J, Bogduk N. The ability of single site, single depth sacral lateral branch blocks to anesthetize the sacroiliac joint complex. Pain Med 2008;9(7):844-50.

35. Gevargez A, Groenemeyer D, Schirp S, Braun M. CT-guided percutaneous radiofrequency denervation of the sacroiliac joint. Eur Radiol 2002;12(6):1360-5.

36. Acevedo JC AA, Casasola O, Chinchilla N, De Giorgis M, Flórez S, Genis MA, et al., editores. Guías para el diagnóstico y el manejo del dolor neuropático. $1^{\text {a }}$ ed. Panamá - Bogotá - Guayaquil: Farmaproyectos latinoamericana; 2009.

37. Dreyfuss P, Michaelsen M, Pauza K, McLarty J, Bogduk $\mathrm{N}$. The value of medical history and physical examination in diagnosing sacroiliac joint pain. Spine (Phila Pa 1976) 1996;21(22):2594-602.

38. Manchikanti L, Pampati V, Fellows B, Baha AG. The inability of the clinical picture to characterize pain from facet joints. Pain Physician 2000;3(2):158-66.
39. Laslett M, Oberg B, Aprill CN, McDonald B. Zygapophysial joint blocks in chronic low back pain: A test of Revel's model as a screening test. BMC Musculoskelet Disord 2004;5:43.

40. Bernard TN, Jr., Kirkaldy-Willis WH. Recognizing specific characteristics of nonspecific low back pain. Clin Orthop Relat Res 1987;(217):266-80.

41. Laslett M, McDonald B, Aprill CN, Tropp H, Oberg B. Clinical predictors of screening lumbar zygapophyseal joint blocks: Development of clinical prediction rules. Spine J 2006;6(4):370-9.

42. Maldjian C, Mesgarzadeh M, Tehranzadeh J. Diagnostic and therapeutic features of facet and sacroiliac joint injection. Anatomy, pathophysiology, and technique. Radiol Clin North Am 1998;36(3):497-508.

43. Manchikanti L, Pampati V, Fellows B, Bakhit CE. Prevalence of lumbar facet joint pain in chronic low back pain. Pain Physician 1999;2(3):59-64.

44. Acevedo JC. Sindrome facetario, revision de la evidencia clinica y experiencia en el Hospital Universitario San Ignacio. xx. [revision]. 2007;2(0123-4048):69-82.

45. Acevedo JC HE, Rodriguez J M, Hakim F, Peña G, Rodríguez A. Enfermedad facetaria lumbar. Análisis clínico de una serie de 37 pacientes con dolor lumbar tratados con bloqueo facetario lumbar. Rev Col de Or Tra 2004;18(3):34-41.

46. Fortin JD, Vilensky JA, Merkel GJ. Can the sacroiliac joint cause sciatica? Pain Physician 2003;6(3):269-71.

47. Grgic V. The sacroiliac joint dysfunction: Clinical manifestations, diagnostics and manual therapy. Lijec Vjesn 2005;127(1-2):30-5.

48. Vermani E, Mittal R, Weeks A. Pelvic girdle pain and low back pain in pregnancy: A review. Pain Pract 2010;10(1):6071.

49. Bogduk N. Pain provocation tests for the assessment of sacroiliac joint dysfunction. J Spinal Disord 1999;12(4):3578.

50. Fortin JD, Falco FJ. The Fortin finger test: An indicator of sacroiliac pain. Am J Orthop (Belle Mead NJ) 1997;26(7):477-80.

51. Hamauchi S, Morimoto D, Isu T, Sugawara A, Kim K, Shimoda Y, et al. Sacroiliac joint dysfunction presented with acute low back pain: three case reports. No Shinkei Geka 2010;38(7):655-61.

52. Kokmeyer DJ, Van der Wurff P, Aufdemkampe G, Fickenscher TC. The reliability of multitest regimens with sacroiliac pain provocation tests. J Manipulative Physiol Ther 2002;25(1):42-8.

53. Laslett M, Aprill CN, McDonald B. Provocation sacroiliac joint tests have validity in the diagnosis of sacroiliac joint pain. Arch Phys Med Rehabil 2006;87(6):874; author reply-5.

54. Laslett M, Aprill CN, McDonald B, Young SB. Diagnosis of sacroiliac joint pain: Validity of individual provocation tests and composites of tests. Man Ther 2005;10(3):207-18.

55. Zelle BA, Gruen GS, Brown S, George S. Sacroiliac joint dysfunction: Evaluation and management. Clin J Pain 2005;21(5):446-55.

56. Mens JM, Vleeming A, Snijders CJ, Stam HJ, Ginai AZ. The active straight leg raising test and mobility of the pelvic joints. Eur Spine J 1999;8(6):468-73.

57. Braun J, Sieper J, Bollow M. Imaging of sacroiliitis. Clin Rheumatol 2000;19(1):51-7.

58. Maigne JY, Boulahdour H, Chatellier G. Value of quantitative radionuclide bone scanning in the diagnosis of sacroi- 
liac joint syndrome in 32 patients with low back pain. Eur Spine J 1998;7(4):328-31.

59. Dreyfuss P, Henning T, Malladi N, Goldstein B, Bogduk N. The ability of multi-site, multi-depth sacral lateral branch blocks to anesthetize the sacroiliac joint complex. Pain Med 2009;10(4):679-88.

60. Cohen SP, Strassels SA, Kurihara C, Crooks MT, Erdek MA, Forsythe A, et al. Outcome predictors for sacroiliac joint (lateral branch) radiofrequency denervation. Reg Anesth Pain Med 2009;34(3):206-14.

61. Kapural L. Sacroiliac joint radiofrequency denervation: Who benefits? Reg Anesth Pain Med 2009;34(3): 185-6.

62. Acevedo JC. Dolor lumbar discogenico. Tratamiento intradiscal con radiofrecuencia. Colombia Dolor 2007;2(16929985):69-82. 